\title{
NOVEL IMMUNOTHERAPEUTIC APPROACH IN GASTRIC CANCER
}

\author{
IN MEMORY OF MY CLOSE FRIEND STEFAN LAZAROV, WHO \\ SUFFERED FROM GASTRIC CANCER AND WAS CURED WITH CIK
}

\author{
M. Gulubova', M. Hadzhi', M. Ignatova', D. Chonov'1, O. Balakan², M. Aydan², J. Ananiev \\ 1Department of General and Clinical Pathology, Medical Faculty, Trakia University - Stara Zagora, Bulgaria \\ ${ }^{2}$ Medical Oncology Department, REYAP Istanbul Hospital - Istanbul, Turkey
}

\begin{abstract}
Gastric cancer (GC) is suitable for immunotherapy because $80 \%$ of it display microsatellite and chromosomal instability, some mutations and DNA hypermethylation. Therefore, GC is more immunogenic. The immunotherapy with monoclonal antibodies, adoptive cell therapy and checkpoint inhibition are discussed. The commonly used monoclonal antibodies are Trastuzumab targeting HER2 and Bevacizumab suppressing VEGF and tumor angiogenesis. Treatment with tumor-specific $T$ cells is called adoptive cell therapy. There is experience with the application of tumor infiltrating lymphocytes (TILs), cytotoxic T lymphocytes (CTLS) and cytokine-induced killer cells (CIK). This review discusses the therapy with innate immune cells with anti-tumor activity such as dendritic cells and NK cells. The checkpoint inhibition was also reviewed. In conclusion, it could be stated that the immunotherapy of GC has the potential to provide a more favorable outcome to patients with GC, but it also have some limitations which need to be considered.
\end{abstract}

Key words: gastric cancer, immunotherapy, antibodies, cell therapy

Corresponding author: Prof. Maya Gulubova, MD, PhD, Department of General and Clinical Pathology, Medical Faculty, Trakia University, 11 Armeiska Street, 6000 Stara Zagora, Bulgaria, tel.: +359664200, e-mail: mgulubova@hotmail.com

T he immuno-oncology is a new field of knowledge about oncology treatment based on the rapidly developing discoveries in the study of immune response and anti-cancer therapies [1].

The immunotherapy is usually combined with chemotherapy, radiation therapy, oncolytic virus therapy or photodynamic therapy [2].

In the base of immunotherapy is the discovery of William Coley in 1893 who has been the first one to observe cancer remission in patients with acute bac- terial streptococcal infections. Later on, Paul Ehrlich in 1909 has supposed that the immune system could prevent cancer development [3].

The immune system is patrolling the body to recognize and destroy pathogens as well as nascent transformed cells and is created to discriminated "self" from "non-self" antigens [4]. Tumor cells appear and are removed by the immune system daily. The ability of the immune system to detect and destroy tumor cells is called "immunosurveillance" [5]. Immune cells 
encounter tumor-associated antigens (TAA) and interact with them in two ways - killings them or tolerating them.

The immunosurveillance is realized by immunoediting [4]. The immunoediting consists in three steps: elimination, equilibrium and escape [4]. During the first elimination step innate and adaptive immune cells gather at the tumor site to attack tumor cells and to remodel the host's stroma that is changed to tumor stroma. The innate immune cells such as natural killer (NK) cells, NK T cells (NKT) and adaptive immune cells CD4+ T cells, CD8+ $T$ cells, all of them secret interferon- $\gamma$ (IFN- $\gamma$ ) that inhibits angiogenesis and tumor cell proliferation. Dendritic cells (DCs) and macrophages also infiltrate the tumor microenvironment (TME) and participate in the anti-tumor immune response (Fig, 1 - adapted from Dunn, 2002 and Ichim, 2015).

After an elimination step, immunoediting enters into an equilibrium step - the longest step. The residual tumor cells exist in an equilibrium with the host's immune system, i.e. DCs and CD8+ T cells maintain tumor cells unresponsive to the signals like IL-12 and IFN- $\gamma$. Tumor cells being genetically unstable change their antigens and escape from immune cells.

In the escape step, there is a suppressive TME that allows tumor cells to escape from the immune system and to begin growing. The recruitment and proliferation of immune cells is suppressed and proliferation of regulatory T cells (Tregs) - FoxP $3+T$ cells occurs (Figure 1). Cancer immunotherapy is pointed to hamper the immunoediting via enhancement of the anti-tumor immune response.

The schematic presentation of immunotherapy approaches is shown in Fig. 2.

Gastric cancer (GC) is suitable for immunotherapy because $80 \%$ of it displays DNA hypermethylation, microsatellite instability (MSI) assocated with mutations in KRAS, ERBB3, and PTEN and chromosomal instability (CIN). Being immunogenic, the majority of GCs may benefit from immunotherapy [6]. GC also has a long latent period and definite premalignant lesions that could be diagnosed and treated.

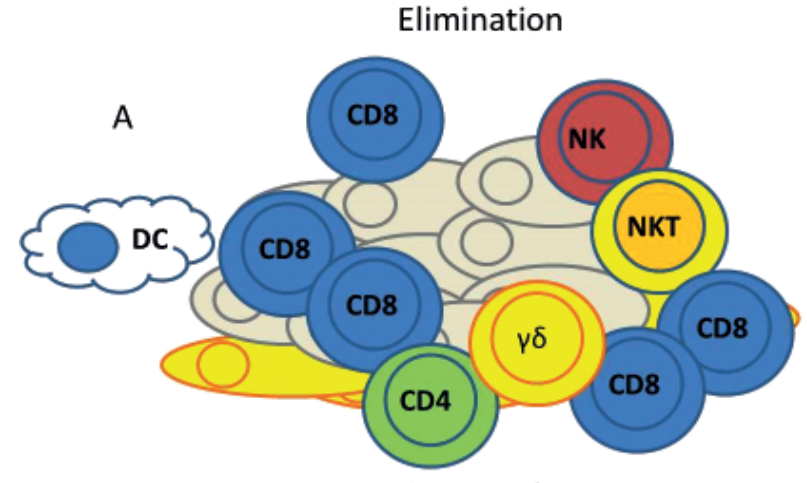

Tumor cell apoptosis/necrosis

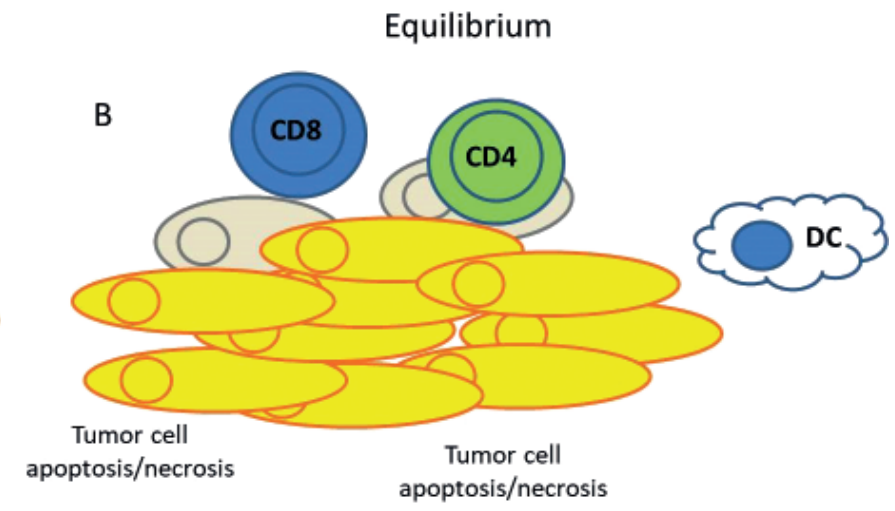

Escape

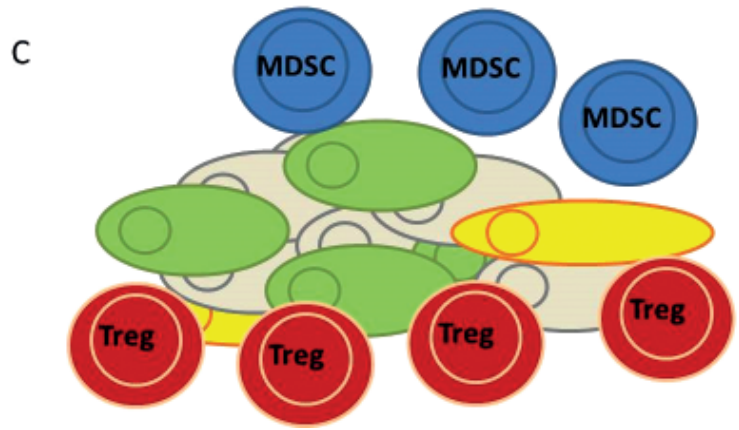

Fig. 1. The immunoediting consists in three steps: elimination, equilibrium and escape. During the first elimination step innate and adaptive immune cells gather at the tumor site to attack tumor cells and to remodel the host's stroma that is changed to tumor stroma. The innate immune cells such as natural killer (NK) cells, NK T cells (NKT) and adaptive immune cells CD4+ T cells, CD8+ T cells, all of them secret interferon-y (IFN- $\mathrm{Y}$ ) that inhibits angiogenesis and tumor cell proliferation. Dendritic cells (DCs) and macrophages also infiltrate the tumor microenvironment (TME) and participate in the anti-tumor immune response . In the escape step, there is a suppressive TME that allows tumor cells to escape from the immune system and to begin growing. The recruitment and proliferation of immune cells is suppressed and proliferation of regulatory T cells (Tregs) - FoxP3+ T cells occurs 


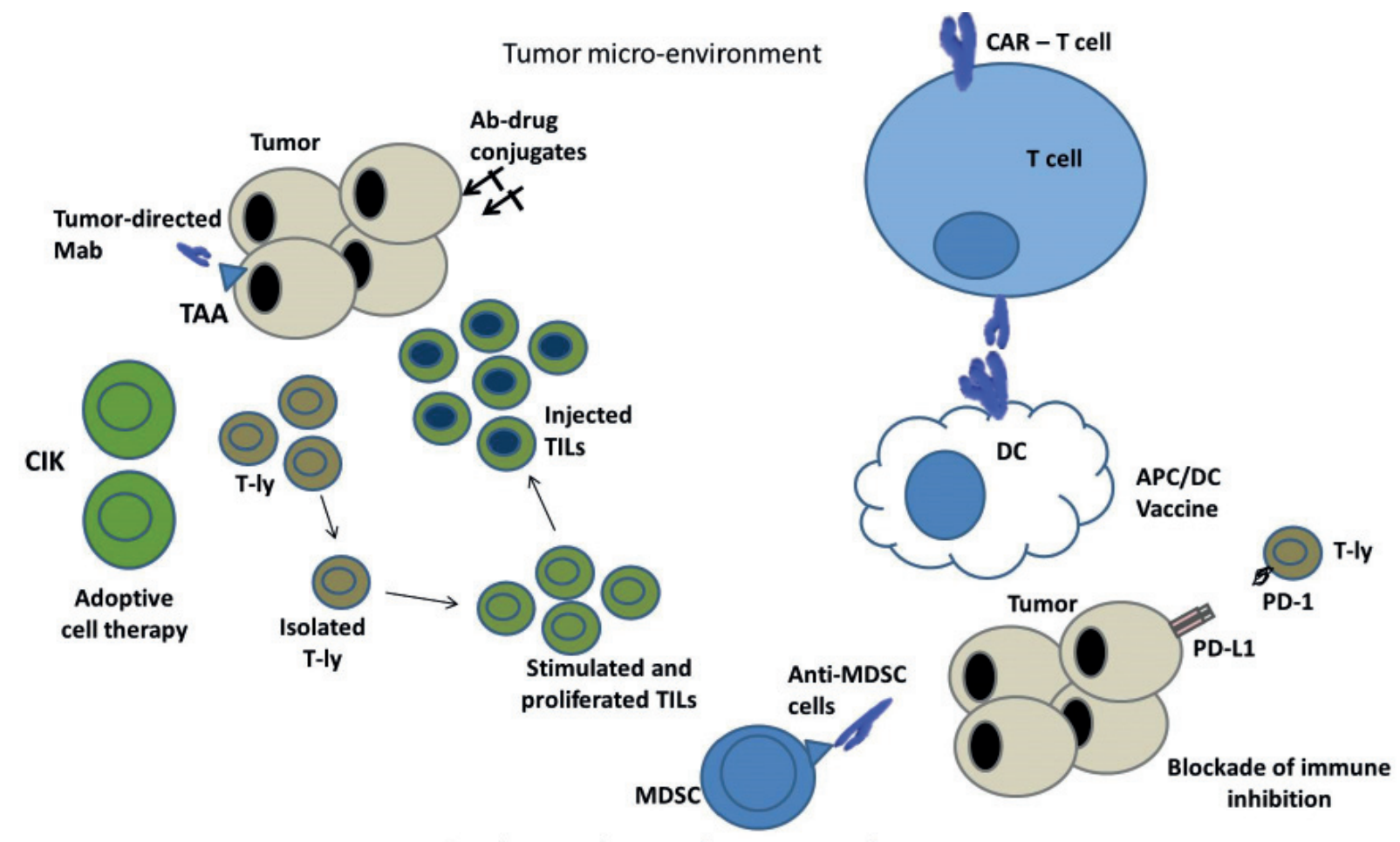

Gastric cancer immunotherapy approach

Fig. 2. The schematic presentation of immunotherapy approaches

\section{THE IMMUNE THERAPY WITH MONOCLONAL ANTIBODIES}

Cancers have defined hallmarks one of which is immunosuppression [7]. Tumors suppress the adaptive immune response down-regulating presentation of TAA and suppressing MHC class I and class II molecules [8]. The TME conducts the expansion of myeloid-derived suppressor cells (MDSCs), that downregulate $T$ cell responses and trigger proliferation of FoxP3+ T cells [8].

The immunotherapy with monoclonal antibodies that enhance immunostimulatory signals or block inhibitory signals have been used to convert TME from immunosuppressive to immunoeffective. The antibodies elaborated have been specific to some TAA and to Fc-y receptor on immune cells such as NK cells or on macrophages. Antibodies may activate the complement system and trigger perforation of the cell membranes [1]. These antibodies are anti-cytotoxic T lymphocyte antigen 4 (CTLA-4) [10], anti-programmed death 1 (PD-1) [11], anti-T cell immunoglobulin and mucin domain-containing protein 3 (TIM3) [12], antiCD40 [13], and anti-OX40 [14].

\section{Trastuzumab (Herceptin-Genentech)}

One of the first elaborated antibodies used in GC therapy is Trastuzumab (Herceptin-Genentech) a humanized antibody that targets human epidermal growth factor receptor-2 (HER2) and suppresses proliferation and survival of HER2-positive tumors [1]. HER2 has been a well-studied member of the epidermal growth-factor tyrosine kinase receptor family. Trastuzumab is an antibody drug conjugate (ADC) a combination between monoclonal antibody and a cytotoxic agent. The antibody against HER2 has been combined with a microtubule inhibitor (DM1) [15]. Moreover, HER2/new expression has been demonstrated in gastric-esophageal junction (GEJ) cancers (mean 22\%, range $0-43$ ). Trastuzumab exerts its effect by preventing dimerization of HER-2, increasing distinction of the receptor and inducing antibodydependent cell cytotoxicity (ADCC) [16]. It is usually combined with chemotherapy (cisplatin/paclitaxel) and radiation [16].

Anti-vascular endothelial growth factor (VEGF) (Bevacizumab)

VEGF is a major regulator of angiogenesis, normal and pathological ones [16]. Increased VEGF expression has been detected in GC and in the serum of GC patients [17]. VEGF exerts its effect via binding to several transmembrane receptors such as VEGFR1 or VEGFR2 [18]. The anti-VEGF therapy has been realized by a humanized anti-lgG1 antibody Bevacizumab (Avastin, Genentech) [19]. Bevacizumab plus chemotherapy has been successfully used in the treatment of colorectal cancer (CRC) [20]. 


\section{Rilotumumab}

The third monoclonal antibody directed against the cmesenchymal-epithelial transcription factor cell surface (c-MET) receptor and its ligand hepatocyte growth factor (HGF) is Rilotumumab [19]. The HGF/MET axis can stimulate the TME and promote angiogenesis.

Currently, the monoclonal antibodies are tumor-directed. These monoclonal antibodies bind to tumorspecific antigens and also bind to definite immune cells. Among these, are bispecific $T$ cell engager (BiTE) and dual-affinity re-targeting (DART) antibodies [15]. One well-known BiTE is Ensituximab (NPC$1 \mathrm{C})$ directed against tumor-specific-variant of MUC5AC, specifically expressed by CRC [21]. AFM13 is a bispecific antibody directed against CD30 and CD16A (found on NK cells) [22].

Another great clone of developing therapy with monoclonal antibodies is the therapy with ADCs. Microtubule inhibitors and DNA-damaging agents have been used in ADCs. The most important aspects of ADCs therapy have been the selection of an appropriate Ag target from the tumor tissue that is not expressed in normal tissue and the selection of a conjugate linker [15].

New antibody-based therapy is the utility of chimeric antigen receptor (CAR) T cells. These are genetically engineered $T$ cell receptors with antibody-based extracellular domain that binds to TAA and a transmembrane portion that activates T cells. Activated CAR T cells initiated cytokine secretion, $T$ cell proliferation, and antigen-specific cytotoxicity. CAR T cells are autologous or allogeneic T cells genetically manipulated by insertion of specific CAR genes via viral vectors [23]. Currently, anti-CD133 CART T cells (CART-133) are utilized to treat patients with advanced epithelial tumors such as hepatocellular carcinoma, CRC, pancreatic carcinoma (NCT02541370). CD133 is expressed by epithelial tumors and CAR-T cells are used against that molecule [24].

\section{ADOPTIVE CELL THERAPY (ACT)}

\section{Therapies with $T$ cells (immune effector cells)}

The treatment with tumor-specific $T$ cells of a cancer patient is called "adoptive cell therapy". The cells in vitro proliferated are lymphokine-activated killer (LAK) cells [25], tumor-infiltrating lymphocytes (TILs) [26], anti-CD3 monoclonal antibody-induced killer cells [27] and cytokine induced killer cells (CIK) [28].

The first trial of ACT in humans utilizing LAK cells has been that of Rosenberg et al., who have treated patients with melanoma with LAK cells plus IL-2 resulting in marked tumor regression [29]. Approximately $30 \%$ of CRC patients and patients with some other cancers treated with LAK cells plus IL-2 also have shown tumor regression [30].

The expanded activated autologous lymphocytes (EAALS) stimulated by anti-CD3 monoclonal antibody (OKT3) and IL-2 in culture and later administered to the patients with GC show improved survival [31]. These are an anti-CD3-antobody-induced activated killers (CD3AK). Activated lymphocytes are developed using an anti-CD3 monoclonal antibody (OKT3) and IL-2, from peripheral blood mononuclear cells (PBMCs) and applied intravenously or intrapleurally [31]. Another method has been developed by isolation of $T$ lymphocytes from cancer patients with immobilizing anti-CD3 monoclonal antibodies. The resulted EAALS (all CD3+ and HLDR+) are shown to be a heterogeneous cell population consisted in CD4+ and CD8+ $T$ cells [31].

TILs are firstly isolated from neoplastic tissue and are cultured in vitro (19). New reports indicate that TIL number positively correlate with patients' survival in CRC [32].

A clinical study of ACT with TILs in combination with chemotherapy in GC resulted in a longer $50 \%$ survival [33]. The majority of TILs have Th1 cytokine profile with high cytotoxic activity against tumor antigens $[16,19]$. Of note, TILs often can promote the expansion of tumor cells since TILs produce IL-17 and IL-21, and TILs could be also Tregs [19]. This fact gives new opportunities for GC immunotherapy application.

ACT with TILs require isolation of T lymphocytes from neoplastic tissue and the activation and expansion of $T$ cells in vitro but the clinical experiences are not satisfactory [33]. The therapy with TILs has some technical difficulties such as isolation of low percent $(30-40 \%)$ T cells from biopsies and also the process of cellular expansion requires about 6 weeks before T cell use [16]. Recently, it has been shown that treatment with TILs isolated from CRC tumor tissue is associated with better survival for CRC patients [32].

Cytotoxic $T$ lymphocytes (CTLs) are isolated from patients' PBMC and induced against GC by survivin peptide [34]. The induced CTLs show specific lysis against tumor cells in vitro [34]. The first clinical tests with adoptive transfer of CTLs have been applied in melanoma patients. Of note, human PBMCs are cultured with IL-2-containing medium in anti-CD3 antibody-coated flasks for 5 days and later are incubated with IL-2 for 9 days. As a result T cells consisted in CD3+ T lymphocytes (97\%) from which $1 \%$ are CD3CD56+, $36 \%$ are CD3+ CD56+, $11 \%$ are CD4+ and $80 \%$ are CD8+ $T$ lymphocytes [21]. This heterogeneous cell population is called $\mathrm{CIK}$, that produce el- 
evated levels of IFN- - , moderate levels of TNF- $\alpha$ and no IL-2 and IL-4. The heterogeneous cell population has a non-major histocompatibility complex (MHC)restricted cytotoxicity and includes expansion of CD3+CD8+CD56- cells to CD56+NKT cells [35, 36]. $\mathrm{CIK}$ cell cytotoxicity is mediated by perforin and is dependent on NKG2D antigen on tumor cells. The CIK cells are able to destroy in vitro the MKN74 tumor cells (a human GC cell line) [21].

On the whole, GC patients treated with CIK are in advanced stage or even with metastatic disease and all have undergone palliate or definitive gastrectomy. CIK is applied usually in combination with chemotherapy with 5-fluorouracil/oxaliplatin or the FOLFOX therapy followed by a decrease of serum levels of tumor markers and the disease free survival rate for the patients has been better in the immunotherapy group than in the control untreated group [37]. The survival rate for patients with GC treated with combination between CIK and chemotherapy has been between 2 and 5 years.

These results explain the appropriate use of $\mathrm{CIK}$ as an ACT in solid tumors [38].

Other in vitro proliferated immune effector cells are DCs [39] and activated NK cells [40].

\section{Dendritic cell vaccines}

The main role of cancer vaccines is to develop TAAspecific $T$ cells. In that way the anti-tumor immune response is enhanced through: i) activation of preexisting immunity; ii) initiation of unprecedented immunity; iii) strengthening the current immune response.

DCs are the most powerful professional antigen-presenting cells (APCs) that bridge innate and adaptive immunity. DCs can activate NK cells, B cells, NKT cells and polarize effector T cells [16].

The use of DCs in clinical trials is limited because of their short life span [41]. For the production of a vaccine DCs are extracted from the patient and incubated with selected TAA and adjuvants [1]. The immature DCs have high phagocytic capacity and are in tumor sites. DCs take up antigens and digest them into small peptides, that are processed and loaded onto MHC class I molecules for presentation to CD8+ $\mathrm{T}$ cells and on MHC class II molecules for presentation to CD4+ T cells.

The monocytes obtained from peripheral blood by apheresis changed in vitro to DCs by the use of cytokines such as GM-CSF and IL-4. The immature DCs are cultured in vitro with TAA and TNF- $\alpha$, IL-1 or IFN-y. Mature DCs then develop and have been injected into the patient [4].
The TAA are antigens that are expressed on tumor cells. For DCs vaccines protein targets isolated from tumor cells are used to stimulate the specific immune response in GC. Such peptides are the tumor-associated antigen HER2/new-derived peptide and melanoma-associated antigen A3 peptide (MAGE-3) [42].

$\mathrm{GC}$ that overexpress HER2/new is treated with vaccination with DCs pulsed with HER2/new peptide and results in tumor regression [4]. Moreover, MAGE-3 peptide used to stimulate anti-tumor immune response in a mouse-model of GC results in cancer regression (43). Autologous DCs expressing human HER2 extracellular and transmembrane domain (AdHER2ECTM) are applied on patients with advanced tumor (colon, breast, ovarian) [44].

Some authors show that DCs number in GC correlated with clinicopathological status and prognosis, where GC patients with increased DC numbers have less lymph node involvement and better survival [45].

The future of DC vaccines is the involvement of genetic modification. Genetic up-regulation of some DC-associated co-stimulatory molecules such as CD40, CD70 and OX40 [46] can enhance the efficacy of future vaccines.

\section{NK cells}

The NK cells are effective in preventing the dissemination of metastatic tumors in humans and the increased NK cells number in some tumors like CRC or GC are indicative of better prognosis. There existed data of NK altered cell phenotype in tumors as the dramatic reduction of their surface marker NKp46. Therefore, NK cells show defects in their ability to degranulate and to produce INF-Y [47]. Interesting data have been reported concerning increased number of apoptotic NK cells (Fas+ NK) in GC patients as compared to normal controls [48].

The key function of NK cells in anti-tumor immune response gives the opportunity to apply them in ACT. However, some obstacles exist that humper the therapeutic use of NK cells, like: i) little data about mechanisms of NK cell function is available; ii) knowledge about different NK cell subsets is scarse; iii) there is a small number of NK cells in the peripheral blood; iiii) different mouse and human NK cell biology; iiiii) obstacles in NK cell stimulated proliferation and activation during manufacturing practice [49].

In regard to $G C$ it is confirmed that large quantities of cytotoxic NK cells can be isolated from PBMC in the presence of K562 cells (having membrane-bound IL15 and 4-BBLigand). The NK cell cytotoxicity is mediated by multiple receptor-ligand interactions that mediated NK cell activation. In conclusion, the ex vivo 
expanded NK cells can be used directly or in combination with monoclonal antibodies in ACT against GC, CRC etc. [19].

Recently, it has been shown that lupeol (a triterpene) favors NK cell proliferation and GC agent.

\section{CHECKPOINT INHIBITION AND CHECKPOINT INHIBITORS}

The dysfunction of CD8+ T lymphocytes and NK cells have been studied in gastrointestinal cancer progression. Programmed cell death protein-1 (PD-1) and cytotoxic T lymphocyte protein-4 (CTLA-4) are important immune checkpoint molecules that down-regulate $T$ cell activation in inflammation and cancer [50]. PD-1 is a co-inhibitory receptor expressed on activated T lymphocytes, Tregs, monocytes and NK cells $[1,50]$. Physiologically, checkpoint inhibitors protect from tissue damage during infections when the immune system is engaged. PD- 1 and CTLA-4 after binding to proper ligands PD-L1/2 or B7, respectively blunt the immune response [4]. PD-1 regulates immune cells after their activation and PD-1 is not expressed on resting and naïve $T$ lymphocytes. While CTLA-4 regulates activation of $T$ lymphocytes [1]. The mechanisms of PD-1 action are similar to that of CTLA-4 and are inhibition of pro-survival and proliferation pathways through the up-regulation of basic leucine zipper transcription factor ATF-like (BATF)

CTLA-4 is also a co-inhibitory molecule expressed on activated $T$ lymphocytes (CD8+ T effector cells) and Tregs. The ligand of CTLA-4 receptor on T lymphocytes binds to B7-1/B7-2 molecule on APCs and inhibits the CD-28-mediated T cell stimulatory signal. The anti-CTLA-4 antibody leads to reactivation and proliferation of $\mathrm{T}$ cells and decrease the recruitment of Tregs in TME [50]. In malignancy, PD-1 (a CD28 family member receptor) on activated $\mathrm{T}$ lymphocytes binds to PD-L1 on tumor cells with subsequent tumor progression [41].

Several studies revealed PD-L1 expression on GC cells. High PD-L1 expression level in GC patients is associated with deeper tumor infiltration, presence of lymph node metastasis, presence of venous invasion, positivity for Epstein-Barr virus infection (EBV+) and positivity for microsatellite instability (MSI) [51]. PD-L1 has been expressed in 50\% on GC cells positive for EBV+ and in $94 \%$ on immune cells. In cases when GC cells are EBV-, the PD-L1+ tumor cells are detected only in MSI patients [52].

Normal gastric mucosa does not express PD-L1 while GC tissue expresses PD-L1 in 42\% of the cases. The high PD-L1 expression is correlated with tumor size, lymph node metastasis and survival [53]. The PD-1 expression on CD4+ and CD8+ TILs is significantly higher than on lymphocytes in the normal gastric mucosa [48].

Recently, several meta-analyses have shown an association between PD-L1 overexpression in GC and worse prognosis for the patients [54].

\section{THE CHECKPOINT INHIBITORS}

Ipilimumab (Yervoy, Bristol-Myers Squibb) is a CTLA-4 inhibitor preventing CTLA-4 interaction with CD80 and CD86. This has been the first checkpoint inhibitor approved by the Federal Drug Association (FDA) [1].

Pembrolizumab (Keytruda, Merck \& Go) is a humanized antibody against PD-1 receptor used in the treatment of melanoma, in non-small cell lung cancer (NSCLC), in breast cancer, GC and CRC [1]. Pembrolizumab has been investigated in a multi-cohort $\mathrm{lb}$ KEYNOTE-012 trial. An overall response rate of $22 \%$ of the patients (36 GC patients) has been reported in this study [55].

Nivolumab (OPDIVO ${ }^{\circledR}$, Bristol-Myers, Squibb) is a humanized antibody against PD-1 receptor the treatment with which results in improvement of the overall survival (OS) in GC patients.

Atezolizumab (Tecentriq, Genentech) is the first FDA approved drug that inhibits PD-L1.

Avelumab is a fully human anti-PD-L1 IgG1 antibody used in a phase lb JAVELIN trial (NCT01772004) [50] that shows good results in the treatment of advanced GC.

Finally, ACT with CIK cells combined with PD-L1/ PD-1 blockade, that enhanced the tumoricidal activity of CIK cells is a promising tool for GC immunotherapy [56].

In conclusion, it may be stated that the immunotherapy for GC has an important clinical application with potential favorable outcome and some limitations. The main problem is the generation of immune effectors and the increasing data about TME interactions must be used to overcome these obstacles in the future.

\section{Conflicts of interest}

The authors declare no conflicts of interest.

\section{Grant information:}

This work was financially supported by the National Science Fund, Bulgaria, Research grant number KP-06-H23/2 from 17.12.2018 


\section{REFERENCES}

1. Kamta J, Chaar M, Ande A, et al. Advancing Cancer Therapy with Present and Emerging Immuno-Oncology Approaches. Front Oncol 2017; 7: a64.

2. Gebremeskel S, Johnston B. Concepts and mechanisms underlying chemotherapy induced immunogenic cell death: impact on clinical studies and considerations for combined therapies. Oncotarget 2015; 6(39): 41600-19.

3. Ichim CV. Revisiting immunosurveillance and immunostimulation: Implications for cancer immunotherapy. J TransI Med 2005; 3(1): 8.

4. Matsueda S, Graham DY. Immunotherapy in gastric cancer. World J Gastroenterol 2014; 20 (7): 1657-66.

5. Burnet M. Cancer; a biological approach. I. The processes of control. Br Med J 1957; 1: 779-86.

6. Nisen P, Weiner GJ, Bokemeyer C. Four subtypes of gastric cancer identified. Cancer Discovery 2014; 4: 1108-09.

7. Cavallo F, De Giovanni $C$, Nanni P, et al. 2011: the immune hallmarks of cancer. Cancer Immunol Immunother 2011; 60: 319-26.

8. Schreiber RD, Old LJ, Smyth MJ. Cancer immunoediting: integrating immunity's roles in cancer suppression and promotion. Science 2011; 331: 1565-70.

9. Gabrilovich DI, Ostrand-Rosenberg S, Bronte V. Coordinated regulation of myeloid cells by tumours. Nat Rev Immunol 2012; 12: 253-68.

10. Leach DR, Krummel MF, Allison JP. Enhancement of antitumor immunity by CTLA-4 blockade. Science 1996; 271: 1734-36.

11. Topalian SL, Hodi FS, Brahmer JR, et al. Safety, activity, and immune correlates of anti-PD-1 antibody in cancer.N Engl J Med 2012; 366: 2443-54.

12. Ngiow SF, Teng MW, Smyth MJ. Prospects for TIM3-Targeted Antitumor Immunotherapy. Cancer Res 2011; 71: 6567-71.

13. Khalil M, Vonderheide RH. Anti-CD40 agonist antibodies: preclinical and clinical experience.Update Cancer Ther 2007; 2: 61-65.

14. Piconese S, Valzasina B, Colombo MP. OX40 triggering blocks suppression by regulatory $T$ cells and facilitates tumor rejection. J Exp Med 2008; 205: 825-39.

15. Marin-Acevedo JA, Soyano AE, Dholaria B, et al. Cancer immunotherapy beyond immune checkpoint inhibitors. J Hematol Oncol 2018; 11: 8.

16. Amedei A, Benagiano $M$, della Bella $C$, et al. Novel immunotherapeutic strategies of gastric cancer treatment. J Biomed Biotechnol 2011; 2011: 437348.

17. Yoshikawa T, Tsuburaya A, Kobayashi $O$ et al. Plasma concentrations of VEGF and bFGF in patients with gastric carcinoma. Cancer Lett 2000; 153: 7-12.

18. Eskens FA, Verweij J. The clinical toxicity profile of vascular endothelial growth factor (VEGF) and vascular endothelial growth factor receptor (VEGFR) targeting angiogenesis inhibitors; a review. Eur J Cancer 2006; 42(18): 3127-39.

19. Niccolai E, Taddei A, Prisco D, et al. Gastric cancer and the epoch of immunotherapy approaches. World J Gastroenterol 2015; 21(19): 5778-93.

20. Hurwitz H, Fehrenbacher L, Novotny $W$ et al. Bevacizumab plus irinotecan, fluorouracil, and leucovorin for metastatic colorectal cancer. N Engl J Med 2004; 350(23): 2335-42.

21. Kim YJ, Lim J, Kang JS, et al. Adoptive immunotherapy of human gastric cancer with ex vivo expanded T cells.Arch Pharm Res 2010; 33: 1789-95.

22. Wu J, Fu J, Zhang M1, et al. AFM13: a first-in-class tetravalent bispecific anti-CD30/CD16A antibody for NK cell-mediated immunotherapy. J Hematol Oncol 2015; 8: 96.
23. Zhang C, Liu J, Zhong JF, et al. Engineering CAR-T cells. Biomark Res 2017; 5: 22.

24. Wang Y, Chen M, Wu Z, et al. CD133-redirected chimeric antigen receptor engineered autologous T-cell treatment in patients with advanced and metastatic malignancies. J Clin Oncol 2017; 35: 3042.

25. Rosenberg SA, Lotze MT, Muul LM, et al. Observations on the systemic administration of autologous lymphokine-activated killer cells and recombinant interleukin-2 to patients with metastatic cancer. N Engl J Med 1985; 313: 1485-92.

26. Rosenberg SA, Spiess P, Lafreniere R. A new approach to the adoptive immunotherapy of cancer with tumor-infiltrating lymphocytes. Science 1986; 233: 1318-21.

27. Yun YS, Hargrove ME, Ting CC. In vivo antitumor activity of anti-CD3-induced activated killer cells. Cancer Res 1989; 49: 4770-74.

28. Rutella S, ludicone P, Bonanno G, et al. Adoptive immunotherapy with cytokine-induced killer cells generated with a new good manufacturing practice-grade protocol. Cytotherapy 2012; 14: 841-50.

29. Rosenberg S. Lymphokine-activated killer cells: a new approach to immunotherapy of cancer.Natl Cancer Inst 1985; 75: 595-603.

30. Rosenberg SA, Lotze MT, Muul LM, et al. A progress report on the treatment of 157 patients with advanced cancer using lymphokine-activated killer cells and interleukin-2 or highdose interleukin-2 alone.N Engl J Med 1987; 316(15): 889-97.

31. Zhang $G Q$, Zhao $H, W u$ JY, et al. Prolonged overall survival in gastric cancer patients after adoptive immunotherapy. World J Gastroenterol 2015; 21(9): 2777-85.

32. Galon J, Costes A, Sanchez-Cabo F, et al. Type, density, and location of immune cells within human colorectal tumors predict clinical outcome. Science 2006; 313: 1960-64.

33. Kono K, Takahashi A, Ichihara F, et al. Prognostic significance of adoptive immunotherapy with tumor-associated lymphocytes in patients with advanced gastric cancer: a randomized trial. Clin Cancer Res 2002; 8: 1767-71.

34. Gang Y, Zhang X, He Y, et al. Efficient induction of specific cytotoxic $T$ lymphocytes against gastric adenocarcinoma by a survivin peptide. Biochem Cell Biol 2012; 90: 701-08.

35. Lu PH, Negrin RS. A novel population of expanded human CD3+CD56+ cells derived from $T$ cells with potent in vivo antitumor activity in mice with severe combined immunodeficiency. J Immunol 1994; 153(4): 1687-96.

36. Franceschetti M, Pievani A, Borleri G et al. Cytokine-induced killer cells are terminally differentiated activated CD8 cytotoxic T-EMRA lymphocytes. Exp Hematol 2009; 37(5): 616-28.

37. Jiang J, $\mathrm{Xu} \mathrm{N}, \mathrm{Wu} \mathrm{C}$ et al. Treatment of advanced gastric cancer by chemotherapy combined with autologous cytokineinduced killer cells. Anticancer Res 2006; 26(33): 2237-42.

38. Sangiolo D. Cytokine induced killer cells as promising immunotherapy for solid tumors.J Cancer 2011; 2: 363-68.

39. Cranmer LD, Trevor KT, Hersh EM. Clinical applications of dendritic cell vaccination in the treatment of cancer. Cancer Immunol Immunother 2004; 53: 275-306.

40. Guo H, Qian X. Clinical applications of adoptive natural killer cell immunotherapy for cancer: current status and future prospects. Onkologie 2010; 33: 389-395.

41. Abozeid M, Rosato A, Sommaggio R. Immunotherapeutic Strategies for Gastric Carcinoma: A Review of Preclinical and Clinical Recent Development. Biomed Res Int 2017; 5791262.

42. Tanaka F, Fujie T, Tahara K, et al . Induction of antitumor cytotoxic $T$ lymphocytes with a MAGE-3-encoded synthetic peptide presented by human leukocytes antigen-A24. Cancer Res 1997; 57: 4465-68. 
43. Yang J , Li ZH , Zhou JJ, et al . Preparation and antitumor effects of nanovaccines with MAGE-3 peptides in transplanted gastric cancer in mice. Chin J Cancer 2010; 29: 359-64.

44. Wood LV, Roberson BD, Agarwal PK et al. Association of autologous AdHER2 dendritic cell vaccination with antitumor activity and number of circulating tumor cells. J Clin Oncol 2017; 35: 3089.

45. Ananiev J, Gulubova MV, Manolova IM. Prognostic significance of CD83 positive tumor-infiltrating dendritic cells and expression of TGF-beta 1 in human gastric cancer. Hepatogastroenterology 2011; 58(110-111): 1834-40.

46. Guo C, Manjili MH, Subjeck JR, et al. Therapeutic cancer vaccines: past, present, and future. Adv Cancer Res 2013; 119: 421-75.

47. Platonova S, Cherfils-Vicini J, Damotte D, et al. Profound coordinated alterations of intratumoral NK cell phenotype and function in lung carcinoma.Cancer Res 2011; 71(16): 5412-22.

48. Saito H, Takaya S, Osaki T, et al. Increased apoptosis and elevated Fas expression in circulating natural killer cells in gastric cancer patients. Gastric Cancer 2013; 16: 473-79.

49. Orange JS, Ballas ZK. Natural killer cells in human health and disease. Clin Immunol 2006; 118: 1-10.

50. Myint ZW, Goel G. Role of modern immunotherapy in gastrointestinal malignancies: a review of current clinical progress. J Hematol Oncol 2017; 10: 86.
51. Gu L, Chen M, Guo D, et al. PD-L1 and gastric cancer prognosis: A systematic review and meta-analysis. PLoS One 2017; 12(8): e0182692.

52. Derks S, Liao X, Chiaravalli AM, et al. Abundant PD-L1 expression in Epstein-Barr Virus-infected gastric cancers. Oncotarget 2016; 7(22): 32925-32.

53. Wu XT, Lui JQ, Lu XT, et al. The enhanced effect of lupeol on the destruction of gastric cancer cells by NK cells. Int Immunopharmacol 2013; 16: 332-40.

54. Xu F, Xu L, Wang Q, et al. Clinicopathological and prognostic value of programmed death ligand-1 (PD-L1) in renal cell carcinoma: a meta-analysis. Int J Clin Exp Med 2015; 8(9): 14595-603.

55. Muro K, Chung HC, Shankaran V, et al. Pembrolizumab for patients with PD-L1-positive advanced gastric cancer (KEYNOTE-012): a multicentre, open-label, phase $1 \mathrm{~b}$ trial. Lancet Oncol 2016; 17(6): 717-26.

56. Dai C, Geng R, Wang C, et al. Concordance of immune checkpoints within tumor immune contexture and their prognostic significance in gastric cancer. Mol Oncol 2016; 10(10): 1551-58.

Received August, 2019 - Accepted October, 2019 